Markus S. Rauscher*, Michael Schardt, Anton J. Tremmel, Michael H. Köhler und Alexander W. Koch

\title{
Nicht-dispersiver Infrarotsensor für die Ölzustandsüberwachung in Verbrennungsmotoren
}

\author{
Non-dispersive infrared sensor for online engine oil condition monitoring
}

\section{DOI 10.1515/teme-2017-0027}

Zusammenfassung: In diesem Beitrag wird ein kompakter optischer Sensor für die Online-Ölzustandsüberwachung in Verbrennungsmotoren vorgestellt. Er basiert auf dem Prinzip der nicht-dispersiven Infrarotspektroskopie (NDIRSpektroskopie) und misst die Absorption in sieben verschiedenen Spektralbereichen. Durch die Kalibierung mit einem linearen Regressionsmodell ermöglicht er die Messung wichtiger Ölzustandsparameter, wie beispielsweise Wassergehalt, Oxidation, Sulfation und Basenzahl. Die Messergebnisse von einem Gebrauchtöldatensatz mit drei verschiedenen Motoröltypen weisen eine gute Übereinstimmung mit Referenzwerten aus einer Laboranalyse auf.

Schlüsselwörter: Nicht-dispersiver Infrarotsensor, optischer Sensor, Ölzustandsüberwachung.

\begin{abstract}
In this article a compact optical sensor for online oil condition monitoring in combustion engines is presented. It is based on the principle of non-dispersive infrared spectroscopy (NDIR spectroscopy) and measures the absorption in seven different spectral regions. By calibrating with a linear regression model, it allows the measurement of important oil condition parameters, such as water content, oxidation, sulfation and base number. The results obtained from a sample data set of three different engine oil types show good agreement with reference values from a laboratory analyis.
\end{abstract}

Keywords: Non-dispersive infrared sensor, optical sensor, oil condition monitoring.

\footnotetext{
*Korrespondenzautor: Markus S. Rauscher, Lehrstuhl für Messsystem- und Sensortechnik, Technische Universität München, Theresienstraße 90 / N5, 80333 München, E-Mail: m.rauscher@tum.de

Michael Schardt, Anton J. Tremmel, Michael H. Köhler, Alexander W. Koch, Lehrstuhl für Messsystem- und Sensortechnik, Technische Universität München, Theresienstraße 90 / N5, 80333 München, E-Mail: m.schardt@tum.de, a.tremmel@tum.de, michael.koehler@tum.de, a.w.koch@tum.de
}

\section{Einleitung}

Motoröle sind im Betrieb hohen Temperaturen, starken mechanischen Belastungen sowie dem Risiko einer Verunreinigung durch feste oder flüssige Fremdstoffe ausgesetzt. Um Schäden durch mangelhafte Schmierung der Bauteile zu vermeiden und um die optimale Leistungsfähigkeit des Motors sicherzustellen, muss der Ölzustand überwacht werden.

Für große Maschinen mit hohem Füllvolumen wird eine regelmäßige Analyse des Öls in Speziallabors durchgeführt. Die dort von Experten vorgenommene Bewertung des Ölzustandes ermöglicht die Anpassung des Ölwechselzeitpunkts an den tatsächlichen Ölzustand. Es entstehen allerdings zusätzliche Kosten für die Entnahme und Analyse der Ölproben. Darüber hinaus bleiben plötzlich auftretende Änderungen des Ölzustands - beispielsweise verursacht durch einen Wassereintritt - zunächst unerkannt, was bereits nach kurzer Zeit zu dauerhaften Schäden führen kann.

Eine Lösung dieser Problematik stellen Online-Sensoren dar, die direkt in der Anlage installiert sind und den Ölzustand kontinuierlich überwachen. Dazu existieren verschiedene Ansätze, die beispielsweise auf Partikelzählung [12], Impedanzspektroskopie [13] oder Viskositätsmessung [9] basieren. Als vielversprechende Messmethoden haben sich auch infrarotspektroskopische Verfahren erwiesen, da sie mit nur einer einzigen Messung Informationen über mehrere relevante Ölzustandsparameter liefern können $[3,4,11,15,17,19-21]$. Dabei erlaubt die Korrelation zwischen Absorption infraroter Strahlung und Konzentration chemischer Verbindungen im Öl eine differenzierte Beurteilung des Ölzustands.

Da sich Fourier-Transform-Infrarotspektrometer, die im Rahmen der Laboranalyse von Motorölen standardmäßig zum Einsatz kommen, aufgrund ihrer Komplexität und Größe jedoch nur bedingt für die Online-Sensorik 
unter rauen Umweltbedingungen eignen, wurde für diesen Anwendungsfall ein Sensor nach dem Prinzip der nichtdispersiven Infrarotspektroskopie (NDIR-Spektroskopie) entwickelt. Durch seinen kompakten Aufbau, der ohne bewegliche Teile auskommt, lässt er sich einfach in den Ölkreislauf integrieren und weist eine hohe Robustheit gegenüber widrigen Umweltbedingungen auf.

\section{Grundlagen der Ölalterung}

In Verbrennungsmotoren kommt es durch die dort entstehenden Belastungen des verwendeten Öls zu einer allmählichen Verschlechterung des Ölzustands. Die Abnahme der Ölqualität kann dabei unterschiedliche Ursachen haben, die getrennt voneinander betrachtet werden müssen. Grundsätzlich wird beim Ölalterungsprozess zwischen Grundölabbau, Addtivabbau und Kontamination durch Fremdstoffe unterschieden.

\subsection{Grundölabbau}

Als Grundölabbau wird die Veränderung der chemischen Struktur des verwendeten Grundöls bezeichnet. Im Fall von Motorölen werden nahezu ausschließlich Öle auf Kohlenwasserstoffbasis eingesetzt, sodass der Grundölabbau von Oxidationsprozessen dominiert wird. Oxidation bezeichnet die chemische Reaktion der Ölmoleküle mit Sauerstoff. Die dabei entstehenden Reaktionsprodukte erhöhen die Viskosität und führen zur Bildung unlöslicher Partikel, die sich ablagern und den Ölkreislauf verstopfen können [14].

\subsection{Additivabbau}

Um Öleigenschaften wie Lasttragfähigkeit, Oxidationsstabilität und Korrosionsschutz zu verbessern, werden Motorölen in der Regel unterschiedliche Additive zugesetzt. Während des Betriebs werden diese Additive langsam abgebaut, wobei sie ihre gewünschte Schutzwirkung entfalten. Additivpakete für Motoröle enthalten häufig Zinkdialkyldithiophosphat (ZDDP) und Calciumcarbonat.

Die Aufgabe des Anti-Wear-Additivs ZDDP besteht dabei im Schutz der Gleitelemente vor Verschleiß und Fressen. Die ZDDP Moleküle reagieren mit der freiliegenden Metalloberfläche und bilden an diesen Stellen eine harte, glasartige Schutzschicht [16]. Darüber hinaus wirkt ZDDP als Antioxidationsmittel, indem es mit Zwischenprodukten des Oxidationsprozesses reagiert und so sein Fortschreiten verhindert.

Calciumcarbonat stellt hingegen die alkalische Reserve des Motoröls dar [16]. Während des Betriebs entstehen durch den Kontakt des Öls mit Verbrennungsgasen und Wasser Säuren, die Korrosion begünstigen und die Oxidation beschleunigen. Das alkalisch wirkende Calciumcarbonat reagiert mit den entstehenden Säuren zu weniger schädlichen Produkten. Die verbleibende alkalische Reserve in Motorölen wird im Rahmen der Laboranalyse durch Titration bestimmt und als Basenzahl (BN) angegeben [18].

\subsection{Kontamination}

Motoröl ist zudem dem Risiko ausgesetzt, im Betrieb mit unterschiedlichen festen und flüssigen Fremdstoffen kontaminiert zu werden. Zu den festen Fremdstoffen zählen dabei beispielsweise Metallpartikel und durch Verbrennungsrückstände eingetragener Ruß. Diese partikelförmigen Verunreinigungen können den Ölfilter verstopfen und führen zu erhöhtem Verschleiß [6]. Die Kontamination durch flüssige Fremdstoffe umfasst unter anderem falsch gewechselte Öle, Kraftstoffeintrag und Wassereintrag. Ein zu hoher Wasseranteil begünstigt Korrosion, vermindert die Schmierleistung und kann durch Hydrolyse zum Zerfall von Additiven führen [7]. Durch den Kontakt mit Verbrennungsgasen gelangen außerdem Schwefeloxide in das Öl, die in Verbindung mit Wasser schwefelige Säuren bilden. Der Gehalt an Schwefeloxiden wird als Sulfation bezeichnet und in der Laboranalyse mittels Infrarotspektroskopie bestimmt.

\section{3 Ölzustandsüberwachung mittels Infrarotspektroskopie}

Die in Kapitel 2 beschriebenen Effekte haben zur Folge, dass sich die chemische Zusammensetzung des Öls mit fortschreitender Alterung verändert. Die im Öl enthaltenen chemischen Verbindungen erfahren dadurch eine Konzentrationsänderung, die mit Hilfe der Infrarotspektroskopie quantifiziert werden kann. Ihr Funktionsprinzip beruht auf dem Zusammenhang zwischen Absorption infraroter Strahlung und Konzentration eines Stoffes und wird durch das Lambert-Beer'sche Gesetz in Gleichung (1) beschrieben. Dabei bezeichnet $A(\tilde{\nu})$ die Absorption der Strahlung bei der Wellenzahl $\tilde{\nu}, c$ die Stoffkonzentration, $\alpha(\tilde{\nu})$ den Extinktionskoeffizienten und $d$ die Schichtdicke der durchstrahlten Probe [10]. 


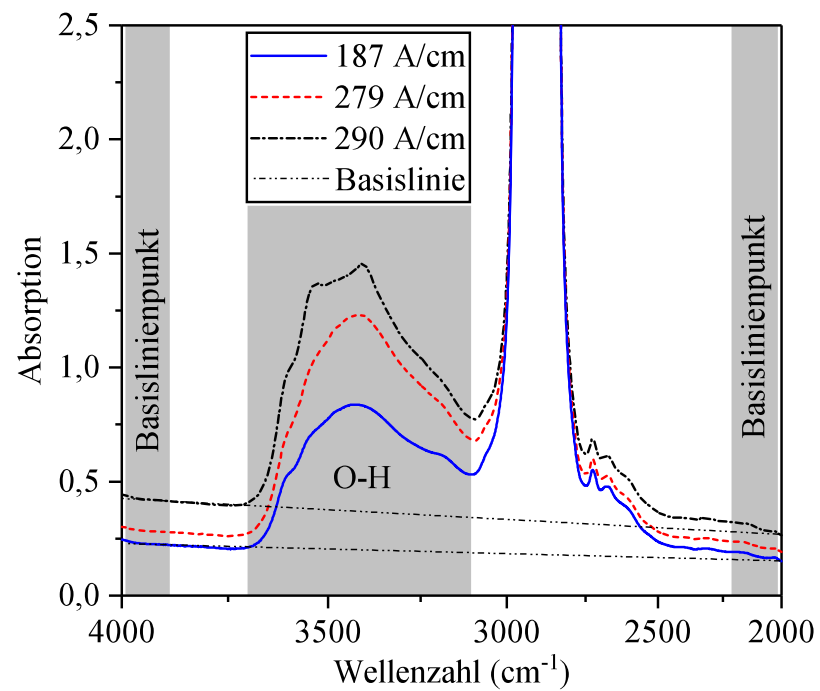

Abb. 1: Absorptionsspektren eines Zylinderöls mit unterschiedlichem Wasser- und Rußgehalt. Durch eine Basislinienkorrektur mit zwei Stützstellen kann der Einfluss von partikelförmigen Verunreinigungen reduziert werden.

$$
A(\tilde{\nu})=-\log _{10}(T(\tilde{\nu}))=c d \alpha(\tilde{\nu})
$$

Die Transmission $T(\tilde{\nu})$ der Probe kann nach Gleichung (2) berechnet werden. $I(\tilde{\nu})$ bezeichnet dabei die gemessene Strahlungsintensität mit in den Strahlengang eingebrachter Probe und $I_{0}(\tilde{\nu})$ die gemessene Strahlungsintensität ohne Probe.

$$
T(\tilde{\nu})=\frac{I(\tilde{\nu})}{I_{0}(\tilde{\nu})}
$$

Im Folgenden wird vor der Vorstellung des konkreten Messaufbaus untersucht, wie sich unterschiedliche Ölalterungseffekte auf das infrarote Absorptionsspektrum auswirken. Dazu werden Absorptionsspektren von real gealterten Motorölproben analysiert, die mit einem FourierTransformations-Infrarotspektrometer aufgezeichnet wurden. Auf Basis der Analyseergebnisse werden anschließend optische Bandpassfilter für die Umsetzung des NDIR-Sensors ausgewählt.

\subsection{Analyse von Gebrauchtölspektren}

Durch die Wellenzahlabhängigkeit des stoffspezifischen Extinktionskoeffizienten $\alpha(\tilde{\nu})$ wirken sich die in Kapitel 2 beschriebenen Alterungsmechanismen auf unterschiedliche Spektralbereiche des Infrarotspektrums aus.

Abbildung 1 zeigt einen Ausschnitt der Absorptionsspektren eines 2-Takt-Schiffsdiesel-Zylinderöls in drei unterschiedlichen Alterungszuständen, wobei die Ölproben

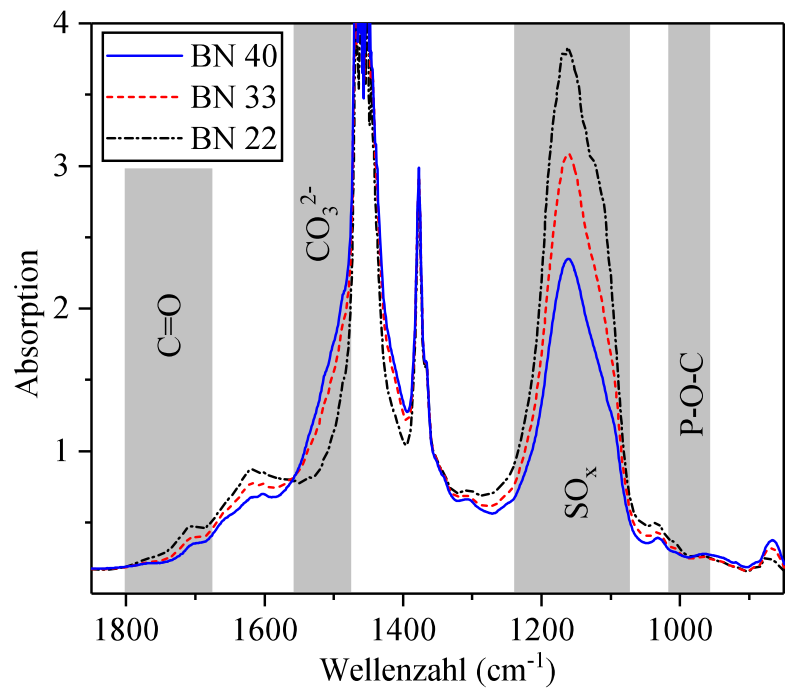

Abb. 2: Absorptionsspektren eines Zylinderöls in verschiedenen Alterungszuständen. Mit zunehmender Alterung (fallende BN) sinkt der Gehalt an alkalischem Carbonat $\left(\mathrm{CO}_{3}{ }^{2-}\right)$, die Öloxidation $(\mathrm{C}=\mathrm{O})$ und die Sulfation $\left(\mathrm{SO}_{\mathrm{x}}\right)$ nehmen hingegen $\mathrm{zu}$.

mit einer unterschiedlichen Menge Wasser und Ruß belastet sind. Der Wasseranteil der Ölproben wurde gemäß der Norm ASTM E2412 [1] bestimmt und weist Werte zwischen $187 \mathrm{~A} / \mathrm{cm}$ (Absorptionseinheiten pro Zentimeter) und $290 \mathrm{~A} / \mathrm{cm}$ auf. Im Infrarotspektrum ist der Wassergehalt im Wellenzahlbereich zwischen $3700 \mathrm{~cm}^{-1}$ und $3150 \mathrm{~cm}^{-1}$ anhand der Sauerstoff-Wasserstoff (O-H) Streckschwingung messbar, die hier eine starke Absorption aufweist [5].

Eine Verunreinigung der Ölprobe durch partikelförmige Fremdstoffe wie beispielsweise Ruß wirkt sich in Form einer Basislinienverschiebung auf das Infrarotspektrum aus. Durch Streuung des eingestrahlten Lichts werden mit steigender Partikelzahl höhere Absorptionswerte gemessen. Die Streueffekte haben bei kürzeren Wellenlängen einen stärkeren Einfluss, sodass eine geneigte Basislinie entsteht. Um diesen Effekt zu korrigieren, muss die Basislinienverschiebung an mindestens zwei Stützstellen gemessen werden. Die Spektralbereiche um $4000 \mathrm{~cm}^{-1}$ und $2000 \mathrm{~cm}^{-1}$ eignen sich dafür, da hier keine Stoffe starke Absorptionslinien aufweisen, die während des Alterungsprozesses eine Konzentrationsänderung erfahren. Somit stellt die Absorption bei $2000 \mathrm{~cm}^{-1}$ ein Maß für den Rußgehalt dar.

Abbildung 2 zeigt das Absorptionsspektrum eines gebrauchten Zylinderöls mit einer verbleibenden BN zwischen $22 \mathrm{mgKOH} / \mathrm{g}$ und $40 \mathrm{mgKOH} / \mathrm{g}$, wobei der Einfluss von partikelförmigen Verunreinigungen durch eine Basislinienkorrektur bereits kompensiert wurde. Zur Messung des Grundölabbaus durch Oxidation wird die infrarote 
Absorption im Wellenzahlbereich zwischen $1800 \mathrm{~cm}^{-1}$ und $1670 \mathrm{~cm}^{-1}$ herangezogen. In diesem Spektralbereich absorbiert die Kohlenstoff-Sauerstoff Doppelbindung $(\mathrm{C}=\mathrm{O})$, die im Rahmen des Oxidationsprozesses entsteht [5].

Die Sulfation kann spektroskopisch im Wellenzahlbereich zwischen $1250 \mathrm{~cm}^{-1}$ und $1080 \mathrm{~cm}^{-1}$ nachgewiesen werden, da in diesem Spektralbereich Schwefeloxide, die in Verbindung mit Wasser zur Bildung von Säuren führen, starke Absorptionsbanden aufweisen [5].

Der Additivgehalt gebrauchter Motoröle ist ebenfalls mit Hilfe der Infrarotspektroskopie bestimmbar. Carbonatverbindungen, die als alkalische Reserve des Öls dienen, weisen starke Absorption im Wellenzahlbereich zwischen $1550 \mathrm{~cm}^{-1}$ und $1400 \mathrm{~cm}^{-1}$ auf [5]. Dieser Spektralbereich überschneidet sich mit einer Absorptionsbande der Kohlenwasserstoffverbindungen des Grundöls, sodass für die Bestimmung des Carbonatgehalts nur der Wellenzahlbereich zwischen $1550 \mathrm{~cm}^{-1}$ und $1480 \mathrm{~cm}^{-1}$ nutzbar ist.

Der Gehalt an Anti-Wear-Additiv ZDDP ist durch die Absorptionsintensität der Phosphor-Sauerstoff-Kohlenstoff (P-O-C) Verbindung im Wellenzahlbereich zwischen $1025 \mathrm{~cm}^{-1}$ und $960 \mathrm{~cm}^{-1}$ messbar [5].

Abbildung 2 veranschaulicht die unabhängige Änderung einzelner Zustandsparameter während des Alterungsprozesses. Mit steigender Beanspruchung des Öls fällt die BN, bedingt durch die Verringerung der Konzentration alkalisch wirkender Carbonatverbindungen. Folglich sinkt die Absorption im Wellenzahlbereich um $1500 \mathrm{~cm}^{-1}$. Mit steigender Beanspruchung des Zylinderöls nehmen auch Oxidation und Sulfation zu, wodurch die Absorption in den entsprechenden Spektralbereichen steigt. Die Konzentration des Anti-Wear-Additivs ZDDP (P-O-C Bande) bleibt im dargestellten Fall hingegen nahezu unverändert.

\subsection{Auslegung der optischen Bandpassfilter}

Die Analyse von Gebrauchtölspektren in Kapitel 3.1 zeigt, dass Wassergehalt, basische Reserve, Anti-Wear-Additivgehalt, Grundölabbau durch Oxidation und Verunreinigung durch Schwefeloxide durch Messung der infraroten Absorption an fünf unterschiedlichen spektralen Banden bestimmt werden können. Rußgehalt und Basislinienverschiebung werden an zwei zusätzlichen Banden erfasst.

Zur Umsetzung des vorgestellten NDIR-Sensors sind somit optische Bandpassfilter notwendig, deren Transmissionsbereiche mit den relevanten Absorptionsbanden übereinstimmen. Um auf speziell angefertigte optische Bandpassfilter verzichten zu können, wurden kommerziell erhältliche Filter ausgewählt, deren optische Eigen-
Tab. 1: Gegenüberstellung von zu messender Zielgröße, entsprechendem Spektralbereich und für die Sensorimplementierung gewählter optischer Bandpassfilter.

\begin{tabular}{lll}
\hline Zielgröße & $\begin{array}{l}\text { Spektralbereich } \\
\mathbf{( \mathbf { c m } ^ { - 1 } )}\end{array}$ & $\begin{array}{l}\text { Bandpassfilter } \\
\tilde{\nu}_{c} / \text { FWHM } \mathbf{( \mathbf { c m } ^ { - 1 } )}\end{array}$ \\
\hline Basislinie & 4000 & $3876 / 75$ \\
Wasser & $3700-3150$ & $3390 / 57$ \\
Basislinie / Ruß & 2000 & $2119 / 40$ \\
Oxidation & $1800-1670$ & $1729 / 27$ \\
BN & $1550-1480$ & $1536 / 24$ \\
Sulfation & $1250-1080$ & $1151 / 27$ \\
ZDDP & $1025-960$ & $980 / 9$ \\
\hline
\end{tabular}

schaften den Anforderungen möglichst gut entsprechen. Tabelle 1 zeigt eine Übersicht der Zielgrößen, Spektralbereiche und ausgewählten Bandpassfilter. Dabei beschreibt $\tilde{\nu}_{c}$ die Wellenzahl maximaler Transmission und FWHM die Halbwertsbreite der Bandpassfilter.

Abbildung 3 zeigt abschließend das Transmissionsspektrum eines Motoröls in frischem und gebrauchtem Zustand. Zusätzlich ist die Transmission sämtlicher im Sensor implementierten Bandpassfilter dargestellt.

\section{Messaufbau}

Abbildung 4 zeigt den prinzipiellen Aufbau des vorgestellten Sensors. Die Messküvette enthält zwei Fenster aus Zinkselenid (ZnSe), die mit Hilfe eines Dichtrings aus Polytetrafluorethylen (PTFE) in einem konstanten Abstand gehalten werden. Eines der Fenster ist mit Bohrungen versehen, durch die die Ölprobe zwischen die Küvettenfenster gepumpt wird. Um die notwendige Druckfestigkeit der Küvetteneinheit zu erreichen, sind die Zinkselenidfenster im Sensorgehäuse mit Hilfe von O-Ringen verpresst. Zwei Dünnschicht-Infrarotlichtquellen (Micro-Hybrid JSIR350) emittieren infrarote Strahlung im Wellenlängenbereich von etwa $2,5 \mu \mathrm{m}$ bis $25 \mu \mathrm{m}\left(4000 \mathrm{~cm}^{-1}\right.$ bis $\left.400 \mathrm{~cm}^{-1}\right)$. Die Strahlung durchdringt die mit Öl gefüllte Küvetteneinheit und wird dabei teilweise absorbiert. Die transmittierte Strahlung trifft anschließend auf zwei pyroelektrische Vierkanal-Detektoren (Infratec LRM-254). Die einzelnen Kanäle der Detektoren sind mit den in Tabelle 1 aufgeführten optischen Bandpassfiltern ausgestattet, um unterschiedliche Spektralbereiche untersuchen zu können. Da die pyroelektrischen Detektoren ausschließlich auf eine Änderung des Strahlungsflusses reagieren, sind die beiden Infrarotlichtquellen gepulst zu betreiben. 


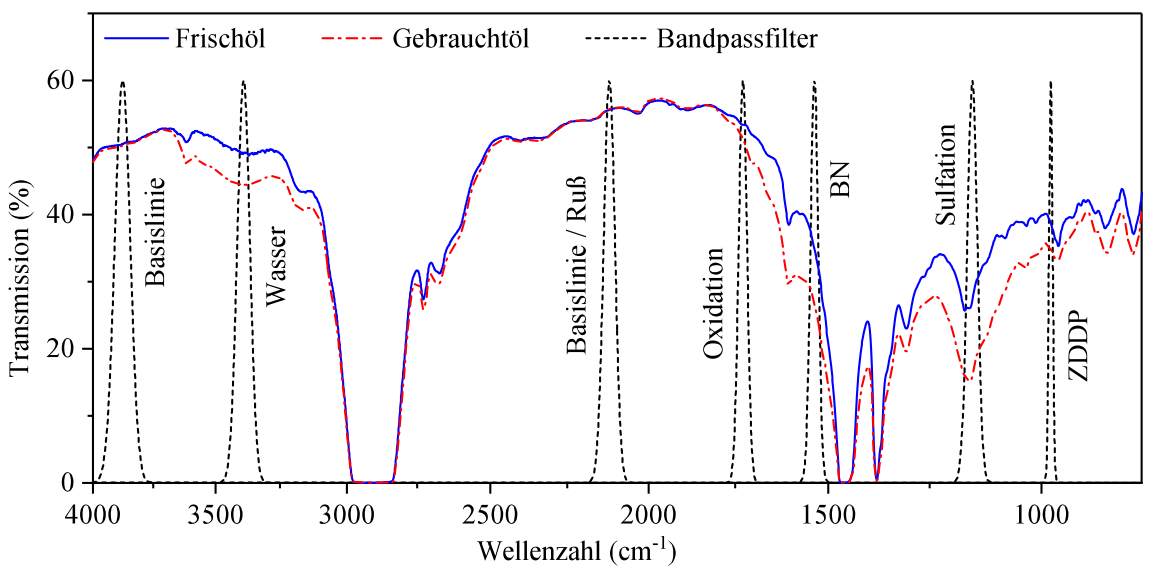

Abb. 3: Transmissionsspektren eines Motoröls in frischem und gebrauchtem Zustand und Veranschaulichung der für den NDIR-Sensor eingesetzten optischen Bandpassfilter.

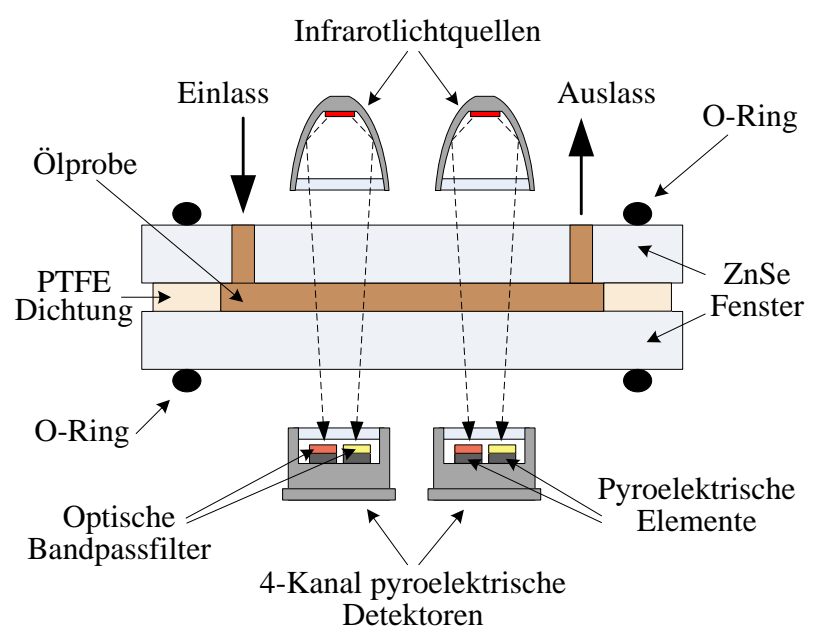

Abb. 4: Funktionsprinzip des vorgestellten nicht-dispersiven Infrarotsensors.

Alle optischen und elektronischen Komponenten sowie die hydraulischen Anschlüsse des Sensors sind in einem robusten Aluminiumgehäuse untergebracht.

\section{Sensorkalibrierung}

Um die Transmission einer Ölprobe nach Gleichung (2) zu messen, muss zunächst der Referenzwert $I_{0}\left(\tilde{\nu}_{c}\right)$ für jeden Kanal des Sensors bestimmt werden. Dafür wird die Küvette aus dem Sensorgehäuse entfernt, um Abweichungen durch Dünnschichtinterferenzeffekte zwischen den Küvettenfenstern zu vermeiden. Anschließend wird die wieder in das Sensorgehäuse eingebrachte Küvette mit einer Ölprobe befüllt und die Intensität $I\left(\tilde{\nu}_{c}\right)$ für jeden Sensorkanal gemessen. Mit Hilfe von Gleichung (1) kann nun die Absorption aus den Messwerten berechnet werden. Um den Einfluss von partikelförmigen Verunreinigungen auf das Messergebnis zu reduzieren, wird die Basislinie der gemessenen Absorptionswerte mit Hilfe des in [15] beschriebenen Verfahrens korrigiert.

Um Wasser- und Rußgehalt, Oxidation, Sulfation, BN und ZDDP-Gehalt aus den gemessenen Absorptionswerten $\mathrm{zu}$ bestimmen, wird im Folgenden ein lineares Regressionsmodell nach Gleichung (3) verwendet.

$$
y=b_{0}+x b_{1}+\epsilon
$$

Das Regressionsmodell besteht hierbei aus dem Skalar $b_{0}$ und dem Vektor $b_{1}$. Es beschreibt den Zusammenhang zwischen dem durch eine geeignete Laboranalyse bestimmten Referenzwert $y$ eines Zustandsparameters und den mit dem Sensor gemessenen Absorptionswerten $x$ einer Ölprobe.

Die Kalibrierung des Sensors erfolgt mit einem Datensatz real gealterter Motoröle. Das Kalibriermodell wird mit Hilfe der multiplen linearen Regression (MLR) erstellt, wobei das Residuum $\epsilon$ durch die Methode der kleinsten Quadrate minimiert wird [10].

Um die Komplexität des Kalibriermodells gering zu halten, sollen nur die Messkanäle des Sensors berücksichtigt werden, die für einen bestimmten Zustandsparameter besonders relevant sind. Das Bias-korrigierte Akaike's Informationskriterium $\left(A I C_{c}\right)$ bietet dabei ein Maß für die Balance zwischen hoher Prädiktionsgüte und geringer Modellkomplexität [8]. Das $A I C_{c}$ wird nach Gleichung (4) berechnet.

$$
A I C_{c}=n \ln \left(\frac{R S S}{n}\right)+\frac{n(n+p)}{n-p-2}
$$




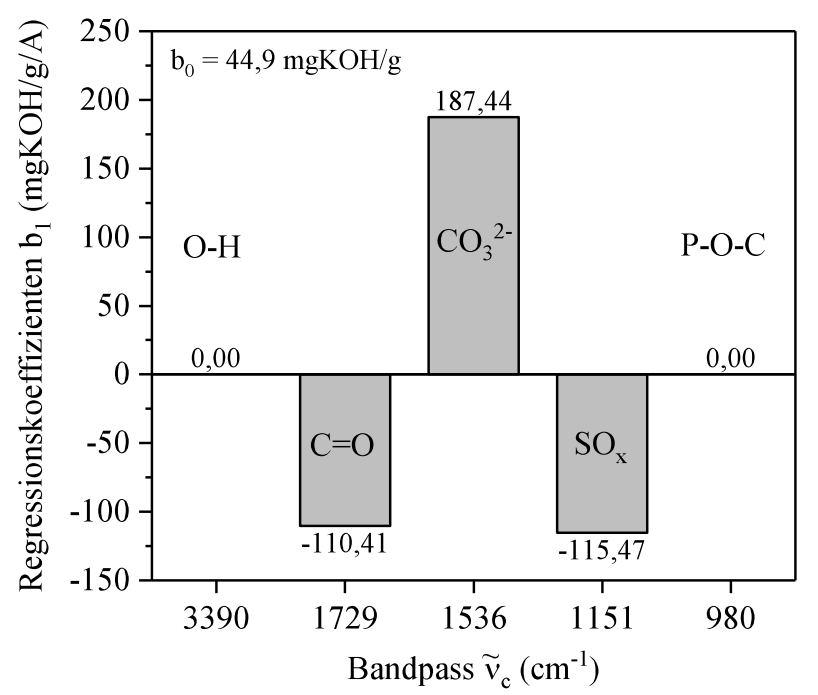

Abb. 5: Grafische Darstellung eines erstellten Kalibriermodells zur Bestimmung der BN eines Zylinderöls.

Dabei bezeichnet $p$ die Anzahl der für das Kalibriermodell verwendeten Messkanäle und $n$ die Anzahl der für die Modellbildung verwendeten Ölproben. RSS bezeichnet die nach Gleichung (5) berechnete Quadratsumme der Residuen von Referenzwerten $y$ und durch das Kalibriermodell vorhergesagten Werten $\hat{y}$.

$$
R S S=\sum_{i=1}^{n}\left(y_{i}-\hat{y}_{i}\right)^{2}
$$

Um die für eine Zielgröße relevanten Messkanäle zu selektieren, werden zunächst Kalibriermodelle für alle möglichen Messkanal-Kombinationen erstellt und $R S S$ und $A I C_{c}$ durch eine vollständige Kreuzvalidierung bestimmt. Das Modell mit dem geringsten $A I C_{c}$ bietet einen Kompromiss zwischen Prädiktionsgüte und Komplexität und wird daher für die Kalibrierung des Sensors ausgewählt.

Abbildung 5 stellt den Vektor $b_{1}$ eines erzeugten Kalibriermodells für die Bestimmung der BN eines 2-TaktSchiffsdiesel-Zylinderöls grafisch dar. Wie aus der Analyse der Infrarotspektren in Abbildung 2 erwartet, ergibt sich eine positive Korrelation der BN mit der Absorption im Spektralbereich der Carbonatbande. Im Kalibriermodell ist der Regressionskoeffizient für den Bandpassfilter bei $1536 \mathrm{~cm}^{-1}$ daher positiv. Gleichermaßen sinkt die BN mit steigender Oxidation und Sulfation des Öls, sodass die Regressionskoeffizienten der Bandpassfilter bei $1729 \mathrm{~cm}^{-1}$ und $1551 \mathrm{~cm}^{-1}$ ein negatives Vorzeichen aufweisen. Der Einfluss der BN auf die Absorption bei $3390 \mathrm{~cm}^{-1}$ und $980 \mathrm{~cm}^{-1}$ wurde im vorliegenden Falls durch das $A I C_{c}$ als zu gering bewertet, sodass diese Messkanäle nicht in das Modell aufgenommen wurden.

\section{Messergebnisse}

Der vorgestellte NDIR-Ölzustandssensor wird nun anhand eines Datensatzes mit drei verschiedenen Motoröltypen evaluiert. Der Datensatz umfasst ein 4-Takt-PKW-Motorenöl (PMO), ein 2-Takt-Schiffsdieselmotor-Systemöl (SSO) und ein 2-Takt-Schiffsdieselmotor-Zylinderöl (SZO). Von jedem Öltyp stehen zwischen 8 und 29 Proben in unterschiedlichen Alterungszuständen mit den dazugehörigen Untersuchungsergebnissen eines Ölanalyselabors zur Verfügung. Die Referenzwerte für Wasser- und Rußgehalt, Oxidation, Sulfation und ZDDP-Gehalt wurden nach ASTM E2412 [1] bestimmt, während die BN nach ASTM D4739 [2] bestimmt wurde.

Alle Ölproben wurden nacheinander mit einer Spritze in die Messzelle des Sensorprototyps injiziert und vermessen. Zwischen den einzelnen Proben wurde die Messzelle mit Reinbenzin gespült, um eine Vermischung der Proben zu verhindern.

Die Kalibriermodelle für alle Zielgrößen und Öltypen werden wie in Kapitel 5 beschrieben durch eine MLR in Kombination mit einer Messkanalselektion nach Akaike erstellt. Zur Validierung der erstellten Modelle kommt eine vollständige Kreuzvalidierung zum Einsatz. Abbildung 6 zeigt die Ergebnisse der Kreuzvalidierung am Beispiel von drei Zielgrößen und Öltypen. Die x-Achse der Diagramme zeigt die im Analyselabor gemessenen Referenzwerte und die $y$-Achse zeigt die mit dem NDIR-Sensor ermittelten Messwerte.

Zur Beurteilung der Modellgüte sind Bestimmtheitsmaß $R^{2}$ und die Wurzel des mittleren quadratischen Fehlers (RMSE) der Modellvalidierung [10] in Tabelle 2 dargestellt. Für die PMO-Proben stehen keine Laboranalysewerte für die BN zur Verfügung, sodass für diese Zielgröße kein Kalibriermodell erstellt wurde. Da in den SZO-Proben nur eine geringe Menge ZDDP enthalten ist, wurde hierfür ebenfalls kein Kalibriermodell erstellt.

Die in Abbildung 6 und Tabelle 2 dargestellten Messergebnisse zeigen einen linearen Zusammenhang zwischen infraroter Absorption und den Ölzustandsparametern entsprechend dem Lambert-Beer'schen Gesetz. Das Bestimmtheitsmaß für den ZDDP- und Rußgehalt des SSO fällt im Vergleich mit den anderen beiden Öltypen niedriger aus. Diese Tatsache ist möglicherweise auf die geringere Varianz dieser Zustandsparameter in den untersuchten SSO-Proben zurückzuführen, wodurch sich zufällige Abweichungen bei der Messdatenerfassung stärker auf das Kalibriermodell auswirken.

Das Kalibriermodell für die Bestimmung der Sulfation des SZO erzielt ebenfalls eine geringere Güte als bei 
Tab. 2: Ergebnisse der Modellvalidierung für alle untersuchten Öltypen und Zielgrößen.

\begin{tabular}{|c|c|c|c|c|c|c|c|}
\hline Öltyp & Parameter & Wasser $(\mathrm{A} / \mathrm{cm})$ & $\operatorname{RuB}(\mathrm{A} / \mathrm{cm})$ & Oxidation $(\mathrm{A} / \mathrm{cm})$ & $\mathrm{BN}(\mathrm{mgKOH} / \mathrm{g})$ & Sulfation $(A / \mathrm{cm})$ & $\operatorname{ZDDP}(\mathrm{A} / \mathrm{cm})$ \\
\hline \multirow{3}{*}{ PMO } & $\mathrm{R}^{2}$ & 0,98 & 0,89 & 0,98 & & 0,99 & 0,91 \\
\hline & RMSE & 0,63 & 0,25 & 0,39 & & 0,56 & 0,42 \\
\hline & Wertebereich & $17,7-31,4$ & $7,66-10,5$ & $12,1-22,9$ & & $13,1-28,7$ & $11,4-16,6$ \\
\hline \multirow{3}{*}{ SSO } & $\mathrm{R}^{2}$ & 0,99 & 0,71 & 0,97 & 0,97 & 0,99 & 0,51 \\
\hline & RMSE & 0,64 & 0,36 & 0,14 & 0,22 & 0,85 & 0,25 \\
\hline & Wertebereich & $13,0-39,9$ & $6,94-9,05$ & $2,09-4,77$ & $9,37-13,2$ & $11,6-36,4$ & $10,0-11,4$ \\
\hline \multirow{3}{*}{ SZO } & $\mathrm{R}^{2}$ & 0,96 & 0,88 & 0,95 & 0,97 & 0,82 & \\
\hline & RMSE & 15,6 & 2,48 & 0,93 & 1,21 & 19,3 & \\
\hline & Wertebereich & $160-394$ & $15,1-33,5$ & $9,41-20,9$ & $34,0-52,4$ & $93,1-198$ & \\
\hline
\end{tabular}

PMO und SSO. Eine mögliche Erklärung dafür ist die starke Absorption von Zylinderöl im Spektralbereich der $\mathrm{SO}_{\mathrm{x}}$-Bande, wie in Abbildung 2 dargestellt. Durch die verringerte Transmission wirken sich auch hier zufällige Messabweichungen stärker auf das Kalibriermodell aus. Im Fall des PMO, für das eine größere Anzahl an Proben vorliegt, wurde bei allen untersuchten Zustandsparametern eine hohe Prädiktionsgüte erzielt.

Für eine vollständige Validierung des Sensors sollten in zukünftigen Arbeiten zusätzliche SSO-Proben mit höherem ZDDP- und Rußgehalt verwendet werden, um den gesamten Zustandsraum dieser Zielgrößen abzudecken. Für eine bessere Bestimmung der Sulfation des SZO bietet sich die Verwendung einer Messküvette mit geringerer Schichtdicke oder die Verwendung eines optischen Bandpassfilters mit größerer FWHM an. Durch diese Maßnahmen würde die transmittierte Intensität erhöht und dadurch den Einfluss zufälliger Messabweichungen minimiert werden.

\section{Zusammenfassung}

In diesem Beitrag wurde ein nicht-dispersiver Infrarotsensor für die Online-Überwachung des Ölzustandes in Verbrennungsmotoren vorgestellt. Durch den robusten und kompakten Aufbau, der ohne bewegliche Teile auskommt, kann der Sensor direkt in einen Ölkreislauf integriert werden. Er basiert auf einem optischen Transmissionsaufbau und ist in der Lage, relevante Änderungen im Infrarotspektrum des Öls mit sieben unterschiedlichen Bandpassfiltern zu messen. Die Bandpassfilter sind so gewählt, dass sie in den Spektralbereichen maximale Transmission aufweisen, die im Laufe der Ölalterung charakteristische Veränderungen aufweisen.

Mit Hilfe einer multiplen linearen Regression und einer Methode zur Selektion relevanter Messkanäle wurden Kalibriermodelle zur Bestimmung des Wasser- und Ruß- gehalts, der Oxidation und Sulfation sowie der Basenzahl und des ZDDP-Gehalts gebrauchter Motoröle erstellt. Die Leistungsfähigkeit des Sensors wurde mit Datensätzen von drei unterschiedlichen Motoröltypen evaluiert. Eine vollständige Kreuzvalidierung der Kalibriermodelle zeigt eine gute Übereinstimmung der Referenzwerte aus einer Laboranalyse mit den Messwerten des NDIR-Sensors.

Durch die quasi-kontinuierliche Messung ist der Sensor in der Lage, plötzlich auftretende Veränderung des Ölzustandes zu erkennen, sodass entsprechende Maßnahmen zur Vermeidung eines Maschinenschadens getroffen werden können. Durch die Beobachtung der Ölzustandsparameter über einen längeren Zeitraum können Ölwechsel bedarfsorientiert ausgeführt und somit Material- und Personalkosten reduziert werden.

Danksagung: Die vorgestellten Arbeiten wurden vom Bundesministerium für Wirtschaft und Energie der Bundesrepublik Deutschland im Rahmen des Förderinstruments ZIM finanziert. Bei unserem Kooperationspartner Comline Elektronik-Elektrotechnik GmbH in Wackersdorf bedanken wir uns für die gute Zusammenarbeit.

\section{Literatur}

[1] ASTM E2412-10, Standard Practice for Condition Monitoring of Used Lubricants by Trend Analysis Using Fourier Transform Infrared (FT-IR) Spectrometry, Annex A1. ASTM International, 2010.

[2] ASTM D4739-11, Standard Test Method for Base Number Determination by Potentiometric Hydrochloric Acid Titration. ASTM International, 2011.

[3] L. Ben Mohammadi, F. Kullmann, M. Holzki, S. Sigloch, T. Klotzbuecher, J. Spiesen, T. Tommingas, P. Weismann, and G. Kimber. A low cost mid-infrared sensor for on line contamination monitoring of lubricating oils in marine engines. Measurement, 7726(0):77260M-77260M-12, 2010.

[4] T. Bley, J. Steffensky, H. Mannebach, A. Helwig, and G. Müller. Degradation monitoring of aviation hydraulic 
(a) SSO - Basenzahl (9 Proben)

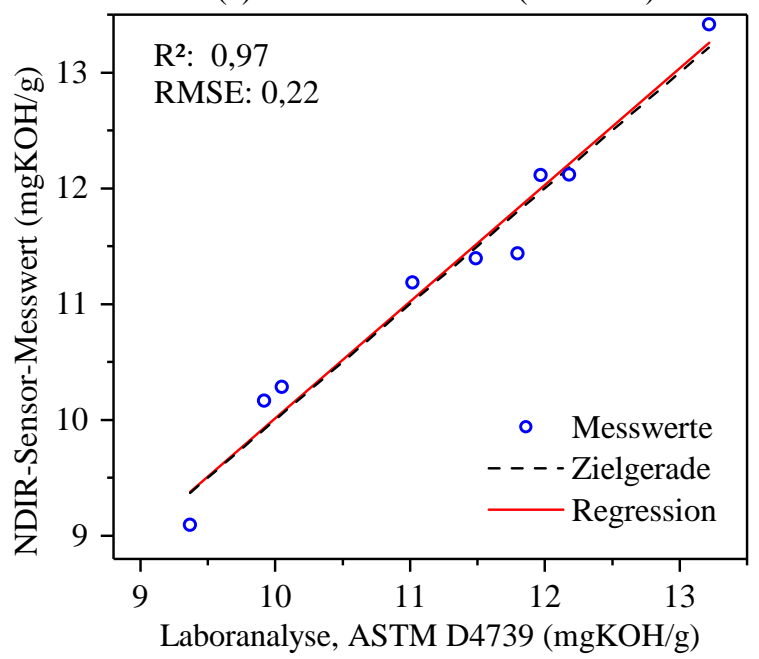

(b) PMO - Oxidation (29 Proben)

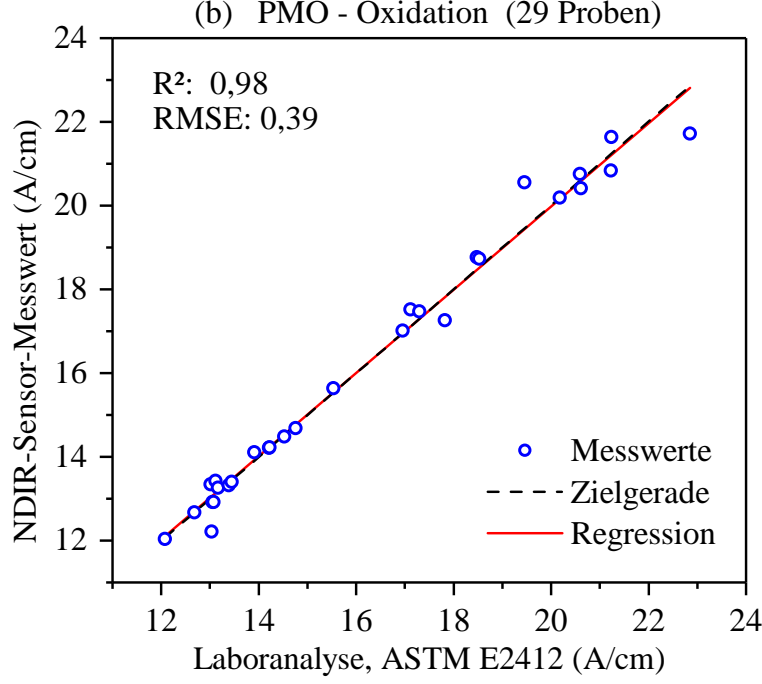

(c) SZO - Wassergehalt (8 Proben)

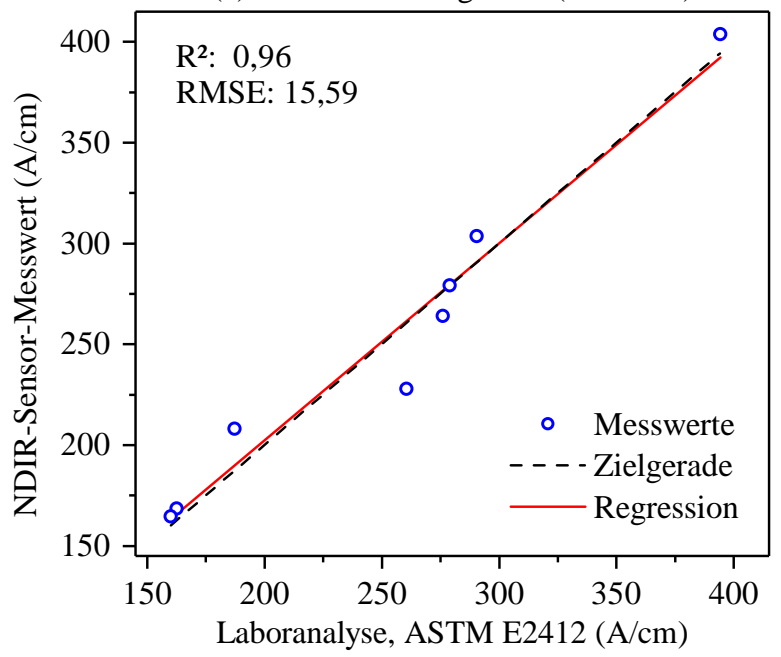

Abb. 6: Ergebnisse der Modellvalidierung für die BN des SSO (a), die Oxidation des PMO (b) und den Wassergehalt des SZO (c). fluids using non-dispersive infrared sensor systems. Sensors and Actuators, B: Chemical, 224:539-546, 2015.

[5] N. B. Colthup. Spectra-Structure Correlations in the InfraRed Region. Journal of the Optical Society of America, 40(6): 397, 1950.

[6] S. George, S. Balla, and M. Gautam. Effect of diesel soot contaminated oil on engine wear. Wear, 262(9-10):1113-1122, 2007.

[7] R. M. Gresham and G. E. Totten. Lubrication and Maintenance of Industrial Machinery: Best Practices and Reliability. CRC Press, 2008.

[8] C. M. Hurvich and C. L. Tsai. Regression and time series model selection in small samples. Biometrica, 76(2):297-307, 1989.

[9] B. Jakoby, M. Scherer, M. Buskies, and H. Eisenschmid. An automotive engine oil viscosity sensor. IEEE Sensors Journal, 3(5):562-568, 2003.

[10] W. Kessler. Multivariate Datenanalyse: für die Pharma, Biound Prozessanalytik. Wiley, 2006.

[11] K. Kudlaty, A. Purde, and A. W. Koch. Development of an infrared sensor for on-line analysis of lubricant deterioration. Proceedings of IEEE Sensors 2003, 2:903-908, 2003.

[12] J. Miller and D. Kitaljevich. In-line oil debris monitor for aircraft engine condition assessment. Proceedings of 2000 IEEE Aerospace Conference, 6(860):49-56, 2000.

[13] A. T. Pérez and M. Hadfield. Low-cost oil quality sensor based on changes in complex permittivity. Sensors, 11(11): 10675-10690, 2011.

[14] D. Pirro, E. Daschner, and A. Wessol. Lubrication Fundamentals, Second Edition. CRC Press, 2001.

[15] M. S. Rauscher, A. J. Tremmel, M. Schardt, and A. W. Koch. Non-Dispersive Infrared Sensor for Online Condition Monitoring of Gearbox Oil. Sensors, 17(2):399, 2017.

[16] L. Rudnick. Lubricant Additives: Chemistry and Applications, Second Edition. CRC Press, 2009.

[17] Spectrolytic GmbH. Marine Engine Market Segment. http:// spectrolytic.com/wp-content/uploads/Spectrolytic-MarineEngine-Market-Segment.pdf. Abgerufen am 08.06.2017.

[18] G. Totten. Handbook of Lubrication and Tribology: Volume I Application and Maintenance, Second Edition. CRC Press, 2006.

[19] F. R. Van De Voort, J. Sedman, R. a. Cocciardi, and D. Pinchuk. FTIR Condition Monitoring of In-Service Lubricants: Ongoing Developments and Future Perspectives. Tribology Transactions, 49(3):410-418, 2006.

[20] B. R. Wiesent, D. G. Dorigo, Ö. Șimșek, and A. W. Koch. Miniaturisierte infrarot-spektrometer zur Ölzustandsüberwachung in offshore-windkraftgetrieben. $t m$ - Technisches Messen, 79(1):73-80, 2012.

[21] B. R. Wiesent, D. G. Dorigo, and A. W. Koch. A miniaturized MID-IR-Spectrometer based on a linear variable filter and pyroelectric line array-Monitoring oil condition. Proceedings IRS² 2013, pages 59-64, 2013. 